\title{
Economics
}

The Open-Access, Open-Assessment E-Journal

Vol. 13, 2019-43 | October 25, 2019 | http://dx.doi.org/10.5018/economics-ejournal.ja.2019-43

\section{Paradigm shifts}

\section{Pierre-André Guy Maugis}

\begin{abstract}
The author studies the evolution of the number of coexisting beliefs in a financial market. Crucially, he undertakes to do so in a framework where the paradigms, beliefs, and models driving agents behavior are left totally unspecified; i.e., the author does not make any parametric or non-parametric model assumptions. The overreaching aim of this exercise is to characterise the dynamic of the variety of beliefs in an auction-based financial market independently of any assumptions on agents behaviors. The resulting framework may be seen as an abstract agent-based model. In a computer experiment the authors exhibits a cycle between two states, so that either all agents act according to the same belief, or there is no leading belief; i.e., there is one dominating belief, or none. Further, the author finds that the frequency of this cycle is positively linked to the quality of the information available to the agents.
\end{abstract}

(Published in Special Issue Agent-based modelling and complexity economics)

JEL G40

Keywords Agent based model; information cascade; herding behavior

\section{Authors}

Pierre-André Guy Maugis, University College London, UK, p.a.maugis@gmail.com

Citation Pierre-André Guy Maugis (2019). Paradigm shifts. Economics: The OpenAccess, Open-Assessment E-Journal, 13 (2019-43): 1-8.

http://dx.doi.org/10.5018/economics-ejournal.ja.2019-43 


\section{Introduction}

We provide an assumption free framework to study the fundamental dynamic of the heterogeneity of beliefs in financial market. We do so by building upon the agent based modeling framework.

From a general abstract perspective, the heuristic of agent based modeling is to first create a copy of a financial market, and then to expriment on this copy to infer properties of a real financial market. The copy is built on two main components: a set of agents and a market structure. Then, as in a real financial market, agents are allowed to interact with each other and the market structure, allowing for experiments to be performed, and phenomenon of interest observed. The finality of this process is that, assuming that the constructed copy is a satisfying replicate of a real financial market, we can draw inference on how events occur in real markets. See for instance Arthur et al. (1996), Huang et al. (2005), Biondo (2018) and LeBaron (2006) for a review.

The market structure component of an agent based model usually replicates a structure commonly found in financial markets, and as such does not raise modeling issues; we will here work on a batch auction market. The agent component and especially how and why agents act and interact is on the other hand a modeling issue. Our view is that as few assumptions or parametric restrictions should be made on this regard as they might be violated by unforeseen behavior and motivations on the part of the agents (Shiller, 1999).

\section{Framework elicitation}

To make minimal assumption regarding agents behavior, we make the following reasoning. We deconstruct the expression of a behavior as a three step process: first information is perceived, then this information is processed, and finally a behavior is expressed. The transformation at the center of this process, that goes from information to behavior, is what could be loosely referred as an agent's belief, but that we define here as an agent's paradigm.

Within this deconstruction, there exists an infinite number of possible paradigms. In its broadest sense, a paradigm may encompass the agent belief in one economic or financial model, his experience of financial markets, his risk appetite, and so on. However, since there exists only a finite number of agents at a given time, we can approximate all currently used paradigms arbitrarily well by $n$ paradigms.

Likewise, this approach allows for an infinite number of different kind of information to be perceived; especially since here the perceived information also contains information that might have been obtained by interacting with other agents. However, for the same reason as above, we can approximate all currently perceived information by $l$ bits of information.

Hence there exists in our framework only $n l$ possible behaviors at a given time. Then, as in an agent based model, each of these behaviors will translate into placing an order at a specific price, or by abstaining to do so. Thus, if we order the $n l$ behaviors, say through a random permutation, we can match the orders in any order book in our framework, and simulate auctions between our agents.

As such auctions take place, agent might change of paradigm. This may be random, or triggered by the model failing to produce a good price. Thus, if an agent's order is far from the auction 
closing price, and he had satisfactory information (to this end we will organize the information bits as satisfactory ones and dissatisfactory ones), he will have a probability $p_{1}$ of changing paradigm, he otherwise has a probability $p_{2}<p_{1}$ of doing so.

We now explore using a computer experiment how the proportion of agents using each paradigm evolves as repeated auctions take place. Note that since our approach is based on an approximation we will in the following only report qualitative results.

\section{Experiment design}

We now formalize the process described above. To do so we will have to distinguish both: between the $n$ different paradigms, and between the $l$ different information bits considered. To this end, we assign to each a number, respectively from 1 to $n$ and from 1 to $l$, and refer to these numbers as paradigm index and information index. The simulation will require a few artifacts parameters, which we will find to have small or no effect on our results, as long as they take reasonable value, as in (Kirman, 1993; Alfarano et al., 2005).

Initialization: To each $N$ agents (this number is static through time, and all $N$ agents remain actors in the market throughout the process), we assign randomly a paradigm index and an information index. The paradigm index is drawn uniformly over $\{1, \cdots, n\}$, while the information index is drawn over $\{1, \cdots, l\}$ using the probabilities $\left\{p_{1}^{i}, \cdots, p_{l}^{i}\right\}$. These last values are parameters of the simulation which we find to have close to no qualitative effect on the ensuing dynamic. We then decide randomly which agents will take part in the next auction, each agent having the same probability $p$ of participating ( $p$ is also a parameter of the simulation that has no effect on the ensuing dynamic.)

Auction: As explained in the introduction, there exists in our approximation $n l$ possible behaviors, which translates into agents having the possibility of putting orders at $n l$ different unknown possible values $v_{1}, \cdots, v_{n l}$. By drawing a random permutation $s$ over $\mathfrak{S}_{n l}$, we can order these values. We now assume that if an agent trades, she trades only one unit. Then we can infer the price that maximizes the number of trades and close the auction: $\forall k \leq n l$ we can compute the number $o_{k}$ of order at the value $v_{k}$, so that the closing price is the price $v_{s\left(k^{*}\right)}$ with $k^{*}$ such that:

$$
k^{*}=\underset{1 \leq k \leq n l}{\operatorname{argmin}}\left\{\left|\frac{N}{2}-\sum_{j=1}^{k} o_{s(j)}\right|\right\} .
$$

Iteration: After the auction, agents are able to determine whether the information they possessed was satisfactory or not, this is made independently of the auction itself. Ignoring the case where the satisfactory bit of information is possibly different for each agent or paradigm, without loss of generality, we will assume that only the bit of information numbered 1 is satisfactory (this is possible since information indices are arbitrary). Then, for each agent we distinguish two cases: either she possessed the satisfactory information that produced $v_{s\left(k^{*}\right)}$, but put an order more than $r$ rank away from it, or not (if an agent placed an order at $v_{s(k)}$, then we say that this order is more 
than $r$ rank away from $v_{s\left(k^{*}\right)}$ if $\left|s(k)-s\left(k^{*}\right)\right| \geq r ; r$ is also a parameter of the simulation which we found to have no qualitative effects on the resulting dynamic for values significantly smaller than $n l$ and $p N$.) In the first case, agents will change of paradigm index with probability $p_{1}$, in the other agents will change of paradigm index with probability $p_{2}\left(p_{2}<p_{1}\right)$. In both cases the new paradigm index is drawn uniformly over all remaining paradigm indexes. Finally, new information indexes are drawn for all agents using the probabilities $\left\{p_{1}^{i} \cdots, p_{l}^{i}\right\}$ as in the initialization.

Remark on $p_{2}$, and the choice of new paradigm: In the simulations we report below, if a paradigm labelled $i$ is a rejected at a given iteration, the paradigm labelled $i$ at a later iteration will be as likely to be selected as any other paradigm. There are several reasons for this choice, but crucially it follows from the fact that agents know that the value of a paradigm highly depends on what is currently the most used paradigm in the market. Therefore, there is little rational to under-select a or over-select a given paradigm if it failed at a previous iteration, especially as the leading paradigm could have changed in between. More to the point, changing the probability does not change the overall dynamic of paradigm shifts; e.g., having agents keep a record of the paradigm that failed to perform and under select them, or vary the value of $p_{2}$ to large values $(\sim 0.25)$, does not appear in our simulations to affect the reported dynamic.

\section{Experiment results}

To present the results we will begin by presenting a representative example of produced dynamic. Then, using this example, we will introduced in more details the two states mentioned above: the homogeneous state where all agents share the same paradigm index and the heterogeneous state where there is no leading paradigm index. Finally, we will compute the probabilities of going from one state to the other for varied values of the parameters.

Representative dynamic example Our focus is the proportion of agents using each of the $n$ paradigms through time. More precisely, using the procedure reported above, we will report the proportion of agent being assigned each paradigm index at each iteration. In Figure 1 we plot these proportions for a simulation of our framework (All computations are done in R (R Development Core Team, 2011).) In this example there are $N=500$ agents, the number of models $n$ is 4 , the number of bits of information $l$ is 5 . The remaining parameters are as follows: the probability of having satisfactory information is $p_{1}^{i}=0.6$, the probabilities of having unsatisfactory information $\left(p_{2}^{i}\right.$ to $p_{5}^{i}$ ) are 0.1 , the probability $p$ of entering a trade is 0.9 and finally $p_{1}=0.9, p_{2}=0.05$, and $r=1$.

In this example the proportion of agents being assigned each paradigm label is behaving differently from iteration 1 to 200 than from iteration 200 to 300. In the first window (1 to 200) there is no leading paradigm label, while in the second window (200 to 300), paradigm label four is consistently concentrating above $80 \%$ of the population. We will call the first state the heterogeneous state, and the other the homogeneous state. In this example the system repeatedly changes of state through time: approximately at iterations 200, 300, 500, 750 and 900 . This type of dynamic is representative of what we obtained for a large number of different parameters, and 


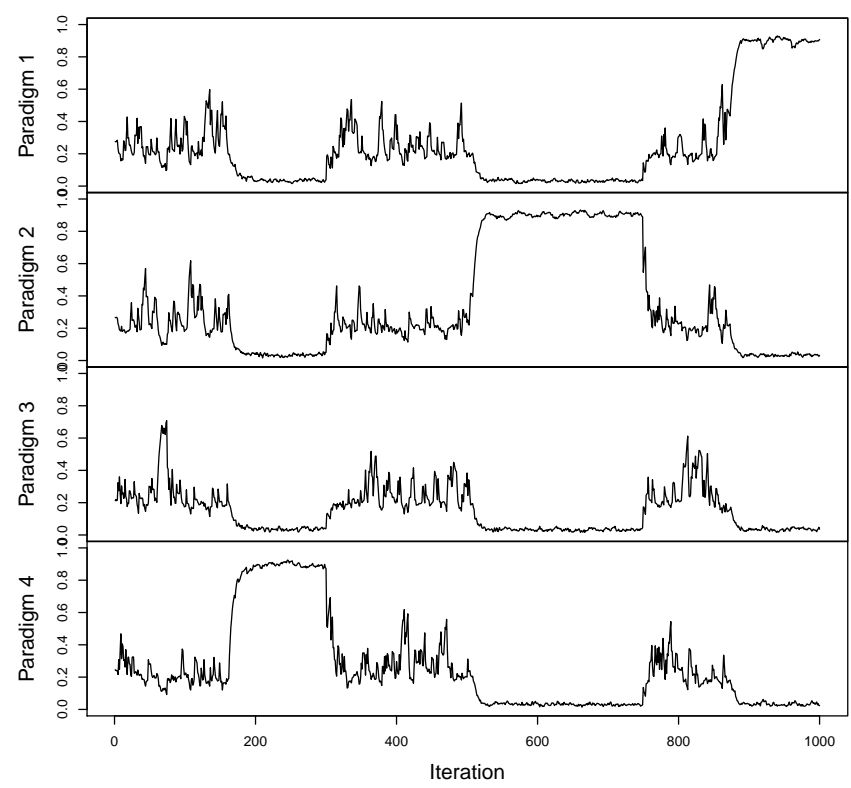

Figure 1: Proportion of agents using each paradigm in an example of 1000 iterations with $n=4$ models, $l=5$ bits of information, $N=500$ agents. The probability of having satisfactory information is $p_{1}^{i}=0.6$, the remaining parameters are $p=0.9, p_{1}=0.9, p_{2}=0.05$, and $r=1$.

slight variation of the framework. It should be noted that such oscillations between two states corresponds to results found in other agent based models (Huang et al., 2005; Huberman and Glance, 1993; Guesnerie, 2002; Kirman, 1993).

Homogeneous and heterogeneous states As the repartition of agents among paradigms at a given iteration is qualitatively well described by the state the system is in-homogeneous or heterogeneous - it is of interest to us to evaluate the probabilities of the system moving from one state to the other. We therefore set $p_{o}$ as the probability of entering the homogeneous state from the heterogeneous state. We likewise define $p_{e}$ as the probability of entering the heterogeneous state from the homogeneous state.

To estimate $p_{o}$ and $p_{e}$, we will simulate a large number of dynamic and average the estimates produced for each dynamic. More precisely, for a dynamic such as the one presented above, we construct from the series shown in Figure 1 an historic of the visited states (we characterize the homogeneous state by one paradigm gathering more than $80 \%$ of the agents for at least two iterations), and then use this historic to estimate of $p_{o}$ and $p_{e}$.

Theoretically $p_{o}$ and $p_{e}$ are functions of all the parameters of the model. However, we report no qualitatively significant link between any of the parameters and these probabilities but for $p_{1}^{i}$, the probability of having satisfactory information. ${ }^{1}$ In our simulations we exhibit a positive link

\footnotetext{
1 Large values of $p_{2}$ (above 0.25 ) can lock the system in the heterogeneous state; however, as $p_{2}$ is essentially the probability that an agent changes paradigm for no market reason, we consider 0.25 large enough to be discarded as a value inconsistent with our proposed framework. We find that in small systems (small $N$ ) having the quantity $n l$ being
} 
$p_{o}$

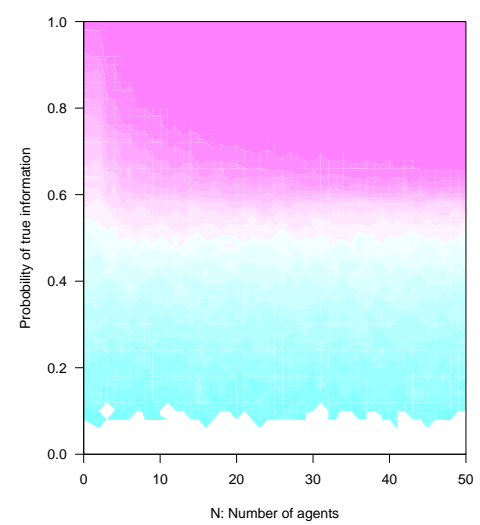

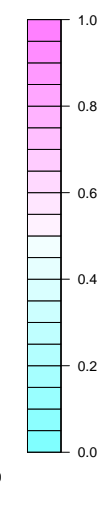

$1-p_{e}$

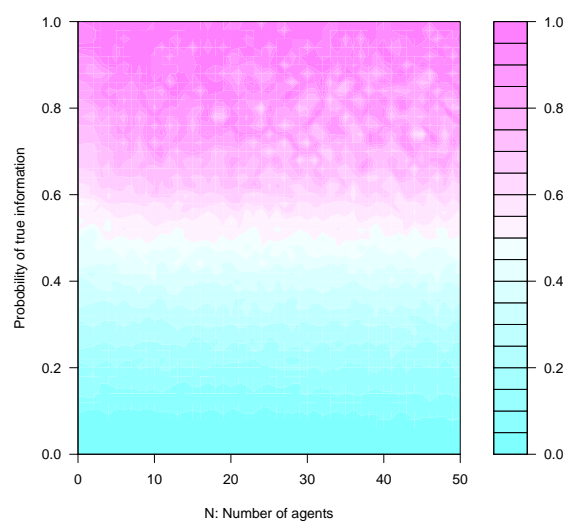

Figure 2: $p_{o}$ and $1-p_{e}$, each point estimated with 500 simulations of 1000 iterations.

between $p_{1}^{i}$ and $p_{o}$ and $1-p_{e}$. In Figure 2 we plotted $p_{o}$ and $1-p_{e}$ as functions of $p_{1}^{i}$ and $N$. We observe that for all $N$ the probabilities are qualitatively similar, but that they increase with $p_{1}^{i}$, the probability of possessing satisfying information.

\section{Discussion}

We observed that independently of all parameters, the system oscillates between two states: a homogeneous one, where all agents use similar paradigms; and a heterogeneous one, where there is no leading paradigm. By its generality, and the simplicity of the framework it is elicited in, this result provides a deeper perspective to similar results found in different agent based models and in laboratory experiments; see Huang et al. (2005); Huberman and Glance (1993); Guesnerie (2002) and Heemeijer et al. (2009).

Further, we find that the quality of the information available to the agents is a key factor that determines the frequency of the oscillations between the two states. We argue that this is surprising, as it implies that better informed agents (agents having a higher probability of possessing satisfactory information), as a group, converge toward the riskier equilibrium. We say the homogenous state is risky as then the stability of market is almost solely dependent on the lead paradigm, and that: i) the lead paradigm is effectively picked at random in our framework (as a paradigm will lead if it is selected by most agents with good information); and ii) the lead paradigm is driving the market (as if for instance the leading paradigm was a different one, say the paradigm labeled 1 instead of 3 , the price dynamic generated might be completely different.) On the other hand the heterogeneous state can be seen as less risky. As the group has a diversified set of used paradigms, heuristically the risk involved in using each paradigm is limited (since it is

close or larger than $N$ may lead to extended heterogeneous states. For computational reasons we could not expand this observation to larger $N$ and $n l$. 
used by a small portion of the population) and might compensate each other (see Huberman and Glance (1993); Easley and O'Hara (2010)).

A possible explanation is that the homogenous state allows to share information efficiently: in the homogeneous state, the system is much simpler (only one paradigm rather than many), and allows agents to communicate more efficiently (as they share the common ground of the having the same paradigm, see Shiller (1995)). Practically, as for instance through the use of implied volatility (Dumas et al., 1998), if 'price=paradigm(information)', and paradigm is known, a price communicates information in as much as paradigm is invertible; i.e., writing 'information $=\operatorname{paradigm}^{-1}$ (price) $)^{\prime}$

\section{References}

Alfarano, S., Lux, T., and Wagner, F. (2005). Estimation of agent-based models: the case of an asymmetric herding model. Computational Economics, 26: 19-49. URL https://doi.org/10.1007/ s10614-005-6415-1.

Arthur, B., Holland, J., LeBaron, B., Palmer, R., and Tayler, P. (1996). Asset pricing under endogenous expectations in an artificial stock market. Santa Fe Institute Publication. URL https://papers.ssrn.com/sol3/papers.cfm?abstract_id=2252.

Biondo, A. E. (2018). Learning to forecast, risk aversion, and microstructural aspects of financial stability. Economics: The Open-Access, Open-Assessment E-Journal, 12: 1-21. URL http: //dx.doi.org/10.5018/economics-ejournal.ja.2018-20.

Dumas, B., Fleming, J., and Whaley, R. E. (1998). Implied volatility functions: empirical tests. Journal of Finance, 53: 2059-2106. URL https://doi.org/10.1111/0022-1082.00083.

Easley, D., and O'Hara, M. (2010). Microstructure and ambiguity. The Journal of Finance, 65: 1817-1846. URL https://doi.org/10.1111/j.1540-6261.2010.01595.x.

Guesnerie, R. (2002). Anchoring economic predictions in common knowledge. Econometrica, 70: 439-480. URL https://doi.org/10.1111/1468-0262.00292.

Heemeijer, P., Hommes, C., Sonnemans, J., and Tuinstra, J. (2009). Price stability and volatility in markets with positive and negative expectations feedback: an experimental investigation. Journal of Economic Dynamics and Control, 33: 1052-1072. URL https://doi.org/10.1016/j.jedc. 2008.09.009.

Huang, H., Wang, D., and Hommes, C. (2005). A robust rational route to randomness in a simple asset pricing model. Journal of Economic Dynamics and Control, 29: 1043-1076. URL https://doi.org/10.1016/j.jedc.2004.08.003.

Huberman, B. A., and Glance, N. S. (1993). Diversity and collective action. In H. Mikhailov (Ed.), Interdisciplinary Approaches to Nonlinear Complex Systems, volume 62. Springer. URL https://doi.org/10.1007/978-3-642-51030-4_5. 
Kirman, A. (1993). Ants, rationality, and recruitment. The Quarterly Journal of Economics, 108: 137-156. URL https://doi.org/10.2307/2118498.

LeBaron, B. (2006). Agent-based computational finance. In L. Tesfatsion, and K. L. Judd (Eds.), Handbook of Computational Economics, volume 2 of Handbook of Computational Economics, chapter 24, pages 1187-1233. Elsevier. URL https://www2.econ.iastate.edu/tesfatsi/hbace.htm.

R Development Core Team (2011). R: a language and environment for statistical computing. $\mathrm{R}$ Foundation for Statistical Computing, Vienna, Austria. URL https://www.R-project.org/.

Shiller, R. J. (1995). Conversation, information, and herd behavior. The American Economic Review, 85: 181-185. URL http://www.jstor.org/stable/2117915.

Shiller, R. J. (1999). Human behavior and the efficiency of the financial system. Handbook of macroeconomics, 1: 1305-1340. URL https://doi.org/10.1016/S1574-0048(99)10033-8. 


\section{Economics}

Please note:

You are most sincerely encouraged to participate in the open assessment of this article. You can do so by either recommending the article or by posting your comments.

Please go to:

http://dx.doi.org/10.5018/economics-ejournal.ja.2019-43

The Editor 